\title{
OVERVIEW ON NEONATAL PALLIATIVE CARE
}

\section{H. Guimarães}

\section{Porto University, Porto, Portugal}

Survival rates for very preterm and critically ill infants are increasing, raising complex ethical issues for health-care providers and parents who face the challenge of making end-of-life decisions for newborns. Palliative care for newborns is holistic and extensive care for an infant who is not going to "get better". It focuses the baby and the family. It may initially be combined with cure-oriented, disease-modifying care and then intensified when that forms of care is no longer helpful or appropriate. The approach is to relieve physical, psychological, social, emotional, and spiritual suffering of the dying baby and family. Opinions and practice regarding end-of-life decisions in neonatal medicine show considerable variations between countries. In Portugal, few studies have examined the circumstances surrounding the last moments of neonates and families in NICUs. The evaluation of care given to neonates and their families in a terminal situation has been improved revealing a more effective pain and distress relief, more spiritual advisor solicitation and free permission of siblings and other relatives to visit. Parental presence during neonatal death was more frequent in the last years. In conclusion, in spite of a neonatal palliative care improvement in last years, we observed that many neonates in end of life still receive curative and aggressive practices. Recommendations and a more consistent and specialized team approach in the care of newborns with a lifelimiting disease are suggested to improve the conditions of the infants and their families. 